\title{
The effect of salt stress on the expression of the brassinosteroid biosynthesis genes
}

\author{
Murgan O.K.*, Efimova M.V. \\ National Research Tomsk State University, Tomsk, Russia \\ * email: reborn_rinni@mail.ru
}

Soil salinization is one of the most unfavorable environmental factors caused by the increased anthropogenic load on the environment. This process is most actively developing on irrigated agricultural soils in arid zones. Excessive sodium chloride content in the soil negatively affects the implementation of many physiological processes in plants, largely due to the generation of reactive oxygen species and the development of oxidative stress. This leads to disruption of the photosynthetic apparatus, a decrease in the intensity of photosynthesis and, as a consequence, a decrease in the productivity of plants; induction of aging or premature death of the plant. Therefore, it is necessary to increase the salt tolerance of agricultural crops. Components of a phytohormonal nature, such as brassinosteroids, are considered as a regulator of plant resistance. Their protective properties under abiotic stress are currently being actively studied. However, the mechanisms underlying the protective effect have not yet been established. We showed the changes in the intensity of the brassinosteroid synthesis genes (CYP450 A90, DET2) expression of Solanum tuberosum (cultivar Lugovskoy) under salinity stress.

Funding: The reported study was funded by RFBR, project number 20-34-90094. 